\title{
La corresponsabilidad educativa y social en el acoso e intimidación escolar. El rol del educador social
}

\author{
Carmen Orte Socías \\ UNIVERSitAT dE LES ILLES BaleARS
}

\section{Resumen}

Este artículo se centra en los comportamientos que adopta el alumnado espectador de situaciones de bullying CON sus iguales. La profundización en las causas de la escasa intervención de los espectadores en estas situaciones está siendo objeto de interés en la investigación. Se pretende formarlos para que adopten un rol más activo, implicándose en comportamientos prosociales de ayuda a sus compañeros víctimas de bullying. La corresponsabilidad como filosofía es una característica que debe tener la intervención. Forma parte de los planteamientos basados en la ayuda a los iguales, en los que el educador social puede tener un papel muy importante.

PALABRAS CLAVE: Acoso e intimidación escolar, Relaciones entre iguales, Ayuda entre iguales, Educación social y escuela, Intervención en bullying escolar, Espectadores de bullying escolar.

\section{Summary}

This paper contains a brief review of the theoretical reference models that explain the causes of bullying at school and specifically focuses on the behaviour of students looking on during these peer-bullying situations. Interest is growing in research into the causes of bystanders' infrequent intervention in these situations. The goal is for students to be educated to take a more active role and get involved in pro-social behaviours to help schoolmates who are victims of bullying. This research should be characterised by coresponsability as a philosophy, since it is an element of intervention approaches based on peer help, in which the social educator has an important role to play.

KEY WORDS: Bullying and harassment in schools, Peer relationships, Peer help, Social and scholastic education, School bullying interventions, Bystanders of school bullying.

\section{Introducción}

Aunque durante muchas generaciones los niños han sido víctimas de otros niños, han sido repetidamente amenazados, agredidos, humillados y rechazados, sólo desde hace algo más de una década empezamos a ser conscientes en nuestro país de que este tipo de comportamientos que ahora conocemos como bullying o maltrato y acoso entre los 
niños y adolescentes es intolerable. Supone una agresión gratuita, cruel y repetida de personas más fuertes o con más poder hacia otras personas más débiles o con menos poder real o percibido. Aunque este tipo de comportamiento es un subtipo de conducta violenta, no ha sido considerado tradicionalmente así. Tampoco ha sido motivo de queja en los centros educativos -por distintas razones-, entre los docentes, los padres o el mismo alumnado que la padece.

Una cuestión importante en este sentido radica en la definición del concepto de violencia. Coincidimos con Batsche y Knoff (1994) en que el concepto de violencia escolar debe definirse de forma amplia para incluir cualquier condición o acto que cree un clima en el que los estudiantes y los profesores sientan miedo o intimidación además de ser las víctimas de asalto, robo, o vandalismo. Incluir el bullying en la definición de violencia amplía de forma importante el análisis sobre sus consecuencias, en las personas que la padecen de forma directa o indirecta, y también en el clima escolar, y en la discusión sobre el tema de la violencia y la seguridad en las escuelas, tradicionalmente más centrada en problemas de asaltos, robos y vandalismo. Sin embargo, según los datos de la investigación sobre incidencia y prevalencia, el bullying puede ser la forma más prevalente de violencia en las escuelas y una de las formas de violencia que es probable que afecten a un mayor número de estudiantes.

El bullying o acoso e intimidación escolar se define como un abuso de poder sistemático, repetido y deliberado. Atenta contra el derecho de los niños y adolescentes a sentirse seguros en un entorno escolar socioafectivo positivo, afectando negativamente a su desarrollo psicológico y social. El acoso, la intimidación y la amenaza forman parte de un continuum de victimización que puede iniciarse con las bro- mas a costa de otro, para ir minándole y socavándole a través de acusaciones, ridiculización, humillación, abuso, caza de brujas y terminar, en algún caso, en muerte por asesinato o suicidio. Este tipo de comportamiento tiene consecuencias importantes para el desarrollo a corto, medio y largo plazo para todas las personas que participan en el mismo. Por ello es muy importante disponer de la información y de las estrategias necesarias para poder prevenirlo y, una vez iniciado, pararlo y ayudar a disminuir sus consecuencias emocionales, físicas y sociales en las personas afectadas.

Uno de los principales resultados de las diversas investigaciones llevadas a cabo sobre el acoso escolar a nivel mundial es, por una parte, el reconocimiento de su prevalencia en las escuelas (Smith et al., 1999; Orte, 2006a), y por otra, sus consecuencias para la salud a corto y largo plazo para las víctimas, quienes padecen esta situación de forma repetida durante una parte importante de su desarrollo.

Las explicaciones sobre las causas de estos comportamientos incluyen aspectos predisponentes de tipo individual, sociocultural y del contexto social de los centros en los que transcurre la vida cotidiana de los escolares. Estas explicaciones han permitido avanzar en los programas y en las prácticas anti-bullying, intentando crear las condiciones que impidan el secretismo en el que se mantiene y refuerza, y generar las estrategias que permitan afrontarlo de forma precoz, minimizando y/o evitando sus peores consecuencias sobre la salud.

Esto ha dado lugar a la puesta en marcha de programas globales; ello incluye estrategias para el conjunto de todos los subsistemas a nivel del centro y de la clase; del profesorado, el alumnado y las familias, algunas veces, en estrategias de trabajo conjuntas. Así, conocer y tomar conciencia 
del bullying, y proporcionar apoyo y formación a las víctimas, trabajar con los agresores y mejorar el clima escolar es el objetivo de una parte importante de estos programas.

Teniendo presentes los modelos teóricos de referencia en las explicaciones sobre las causas del bullying, y sin perder de vista que las intervenciones en el acoso escolar deben afectar a todos los subsistemas implicados de una forma corresponsable; por su importancia y actualidad, nos vamos a referir de forma más concreta a la influencia de los grupos de iguales en el proceso de acoso e intimidación escolar. Las relaciones entre iguales son indicadores importantes de la salud mental del alumnado y predicen su ajuste psicosocial. Por ello es importante ocuparse de estas relaciones y también de:

a) Prestar atención al contexto social en el que se producen estos comportamientos, que puede ser más posibilitador o menos. b) Prestar atención a los grupos a los que el alumno o alumna pertenece o no.

c) Prestar atención a los comportamientos de aprobación o desaprobación de quienes contemplan el acoso (Orte, 1996 y 2005).

Los comportamientos que adoptan los espectadores en situaciones de bullying han despertado mucho interés en las propuestas de intervención en la escuela primaria y secundaria dirigidas a este alumnado. La profundización en las causas de la escasa intervención de los espectadores, entre otras: la ausencia de normas y de comportamientos de apoyo en el contexto escolar, el temor a ser también victimizado, la valoración negativa de las víctimas o la falta de estrategias eficaces para intervenir, ha dado lugar al desarrollo de estrategias de apoyo a los iguales dirigidas a los espectadores, en concreto, a quienes observan el bullying, y desearían tener una parte más activa en su prevención. Los espectadores están siendo objeto de interés en la investigación, con la finalidad de formarles para que adopten un rol más activo. El objetivo es implicarlos en comportamientos prosociales de ayuda a sus iguales víctimas de bullying. Con esa finalidad se ha desarrollado y evaluado un conjunto de acciones destinadas a fomentar el potencial de apoyo y ayuda del alumnado que, aunque no está de acuerdo con los agresores, no interviene para ayudar a las víctimas. Es en este contexto en el que se forma y se da apoyo a este alumnado para que pueda ofrecer distintos tipos de apoyo, ayuda y colaboración a otros en situaciones de vulnerabilidad, como es el caso del bullying. Se trata de proporcionar el apoyo social necesario a nivel estructural y funcional, para que el alumnado víctima de bullying, participe de las redes sociales de ayuda, mejore la calidad de sus relaciones con sus iguales, y poder prevenir y/o reducir el impacto de estas conductas negativas.

\section{Etiología y acoso escolar}

\subsection{Los modelos explicativos}

En algunas ocasiones y debido a la alarma social generada en los medios de comunicación, algunas de las acciones que se emprenden en relación al acoso escolar están más orientadas a justificar una intervención, por ejemplo, disponiendo de un protocolo de detección o de un sistema de penalización más o menos establecido, que en llevar a cabo propuestas eficaces elaboradas a partir de modelos teóricos de referencia en relación a las causas que han podido generar el problema. Aunque no los excluyan, son estrategias, programas y actividades de mayor calado que cumplimentar protocolos o establecer sanciones. No hay una única razón de por qué algunos chicos y chicas se convierten en agresores o en víctimas, más bien un conjunto de variables familiares, es- 
colares, sociales, culturales y personales que pueden prevenir o acrecentar los comportamientos de bullying. Algunos autores (Soutter y McKenzie, 2000) consideran que el determinante más importante de la conducta de bullying es el contexto en el que ocurre.

De hecho y de acuerdo a las aportaciones de Rigby (2003a) sobre esta cuestión, las explicaciones sobre las razones del por qué se producen estos comportamientos de abuso de poder entre escolares (que dan origen a la variedad de programas de intervención) son de distinto tipo y no necesariamente contradictorias entre sí.

El acoso e intimidación entre escolares, el bullying, es una conducta natural entre los niños y niñas que a medida que se desarrollan tiende a desaparecer. De acuerdo con esta teoría, es posible que este tipo de comportamiento forme parte del modo en que los niños pretenden afirmarse a costa de los demás para establecer su dominancia social. Esta teoría da la razón en parte a quienes opinan que el bullying es un comportamiento normal ${ }^{1}$. Este planteamiento tiene en cuenta además el tipo de agresiones que se llevan a cabo a lo largo de este recorrido del desarrollo desde la educación primaria hasta la educación secundaria: el tipo de agresiones, desde las más físicas en primaria a las más verbales y a las formas más indirectas en secundaria. En cualquier caso y en términos educativos, este enfoque es interesante desde el punto de vista de enseñar estrategias de resolución de problemas. Lo importante es que los centros asuman la enseñanza de estrategias de resolución de problemas que puedan ser eficaces para agresores y víctimas, dentro de los currículos adaptados al nivel educativo. También es importante tener en cuenta que este tipo de estrategias coercitivas en las relaciones pueden ser más prevalentes o menos dependiendo de la heterogeneidad del contexto en la composición del alumnado, de las familias y del profesorado, y de las estrategias organizativas para dar respuesta a las necesidades surgidas en los mismos. El contexto puede ser más posibilitador o menos, dependiendo de políticas y programas más amplios de no tolerancia a la violencia y al acoso, elaboradas para dar respuesta a este tipo de necesidades

Un segundo grupo de explicaciones bastante generalizado sobre las causas del acoso y de la violencia escolar entre iguales atribuye este tipo de comportamientos a las diferencias individuales. Establece una relación entre determinadas características de inteligencia social y personalidad y el tipo de comportamientos que se llevan a cabo en una situación de acoso. El alumnado que agrede y se burla de otros de forma repetida, como sistema, como forma de relación, presenta niveles bajos de empatía hacia los demás e inclinaciones hacia la agresividad y el psicoticismo (Slee y Rigby, 1993). Por su parte, el alumnado víctima de otros presenta problemas de introversión, baja autoestima y falta de habilidad en las relaciones sociales (Rigby, 2002). Las causas que han dado origen a este tipo de perfiles y características de unos y de otros podrían atribuirse tanto a influencias genéticas como a condiciones sociales adversas a las que se haya podido estar expuesto en el hogar (Orte, 2006b). En el caso de las víctimas, además, al proceso de deterioro que supone el hecho de estar sometido a una situación de acoso continuado (Orte, 2006a).

Atribuir las causas del acoso e intimidación escolar a problemas individuales presenta algún inconveniente importante. En concreto, se deja de lado la responsabilidad e influencia del contexto escolar en el desarrollo y expresión o no de estos comportamientos negativos. Ello puede influir sin duda en la percepción de responsabilidad sobre el problema y de corresponsabilidad en su solución.

Con todo y dada la importancia para el 
ajuste psicosocial de las relaciones positivas del alumnado con sus iguales, es necesario conocer las características comentadas en el alumnado agresor y/o víctima, o incluso el conocimiento de las situaciones adversas que pueden estar reforzando una determinada forma de relacionarse negativamente con los demás. Es una información que debe utilizarse para guiar las acciones educativas en los centros, diseñar los programas de intervención con el alumnado agresor ayudándole a controlar y canalizar de forma más adecuada la cólera y la agresividad; diseñar los programas dirigidos al alumnado más vulnerable a la agresión a través de programas de formación y práctica de habilidades sociales; y orientar y formar a las familias en estas situaciones.

Siguiendo con el esquema aportado por Rigby (2003a), otro gran grupo de explicaciones sobre las causas del bullying se centra en el rechazo a las diferencias como elemento de presión entre las personas y los grupos, amparados bajo la diferencia de poder histórica y en muchos casos compartida social y culturalmente, por ejemplo, en cuestiones de género, etnia, raza o grupo social de pertenencia. Quienes pertenezcan a los grupos más desfavorecidos y/o minoritarios, percibidos con menor poder y valorados de forma negativa, tendrán más posibilidades de ser maltratados según este análisis. Estas explicaciones se basan en el análisis del perfil de las personas maltratadas. En concreto y en relación al género, los datos epidemiológicos de los estudios sobre bullying señalan la mayor probabilidad de que sean los chicos quienes lleven a cabo comportamientos considerados como acoso e intimidación, antes que las chicas (Olweus, 1993; Smith y Sharp, 1994; Orte et als. 2004). Además de los datos epidemiológicos sobre el rol del género en la mayor frecuencia del bullying masculino, los enfoques educativos del maltrato y de las desigualdades por razones de género desde esta perspectiva sociocultural, así como los elementos conceptuales que comparten la violencia de género y la violencia entre iguales, recomiendan el tratamiento de estos dos problemas de forma conjunta en los currículos escolares (Orte, 2002, 2005).

De todas maneras y dejando a un lado la cuestión del género citada, aunque no se disponga de suficiente apoyo empírico en la importancia de otros aspectos culturales como, por ejemplo, la clase social, esta perspectiva pone en evidencia la importancia de las diferencias, especialmente aquellas que han sido señaladas como "causa" de agresión en los estudios epidemiológicos sobre bullying (Rigby, 2003a).

El tratamiento preventivo de los prejuicios y de la discriminación de las diferencias puede enmarcarse en este enfoque sociocultural. En los contenidos, en la organización y en las metodologías de enseñanza basadas en el aprendizaje de la empatía, la resolución de conflictos, el trabajo cooperativo o el pensamiento crítico, como formas de influir en la comprensión y aceptación, el respeto a las diferencias socioculturales, y en las relaciones positivas entre iguales.

\subsection{La influencia del contexto y del grupo}

Uno de los enfoques que mayor investigación y propuestas de intervención están generando es el enfoque centrado en la importancia e influencia del contexto y del grupo de pertenencia como causa del bullying y de otros problemas de conducta (Rulison et als.; Dishion et als., Nylun, en Society for Prevention Research, 2007). Un enfoque que concibe los comportamientos de bullying en un determinado contexto social con dos niveles de influencia que pueden ser objeto de diagnóstico y de intervención. Por una parte y a escala más 
global, las políticas y los programas que se llevan a cabo en un centro educativo concreto que presten más atención o no al fomento de las relaciones entre los miembros de la comunidad en general y de los estudiantes y sus familias en particular. Por otra parte, el nivel de influencia del grupo de iguales a los que el alumnado pertenece y con los que comparte vínculos e intereses en su vida diaria. Aunque de manera distinta, ambos grupos influyen de forma muy importante en las percepciones y en el comportamiento de sus miembros. En el caso de los grupos de referencia del alumnado, pertenecer o no a ellos puede convertirse en un momento determinado en una amenaza. El motivo puede ser el resentimiento contra alguien que se piensa o se imagina que ha cometido un acto equivocado o desleal. Estaría dentro de este grupo el caso del alumno español que se suicidó tras haber sido sometido a acoso continuado por parte de su grupo de referencia y de amistad, que más tarde se convirtió en su enemigo. Por otra parte, y desde otro punto de análisis, en el grupo se pueden llevar a cabo acciones contra otros. De hecho, los estudios epidemiológicos indican que a nivel grupal se lleva a cabo una parte importante de las acciones de acoso e intimidación a otros (Orte et als., 1997-2000; Defensor del Pueblo, 2007). Se considera que una parte muy importante de los actos de bullying se mantienen por la conexión con un grupo, antes que por motivos individuales. En cualquier caso, la dimensión grupal del fenómeno no es una cuestión sin importancia, porque, tanto si se genera y se mantiene desde el grupo de pertenencia como si los comportamientos se llevan a cabo por una o varias personas, apoyados de forma pasiva por otros, la dimensión grupal estaría también presente. Aunque los protagonistas principales sean el agresor y la víctima, en situaciones de acoso todo el alumnado tiene un papel en el mismo ya sea como agresor, seguidor, partidario, espectador, posible defensor, defensor, etc. (Salmivalli, 1999; Olweus, 2001).

La implicación para los centros educativos es que deben ser conscientes de los roles que juegan los grupos y los individuos. Deben identificar los grupos y trabajar con ellos. Algunos enfoques de intervención grupal más desarrollados están diseñados para parar estos comportamientos. Tienen su fundamento en una dimensión grupal más o menos establecida y en los roles que ejercen los miembros del grupo. Dichos roles tienen distinta importancia en el mantenimiento o cesación de los comportamientos de bullying. El método de no-culpabilización de Maines y Robinson (1992) y el método Pikas (2002) parten del reconocimiento no-culpabilizador del sufrimiento de la víctima y pretenden una búsqueda constructiva de soluciones por parte de los implicados (tanto agresores como víctimas) que les comprometa con su propia conducta.

A partir de los modelos teóricos comentados, a los que nos hemos referido como no excluyentes entre sí, se han generado la mayoría de estrategias de intervención que en este momento se están utilizando en relación a los comportamientos de acoso e intimidación entre escolares en los centros educativos. En muchas ocasiones, acompañados de materiales didácticos dirigidos al alumnado y a las familias, y de sistemas de denuncia de acoso presenciales $y / o$ virtuales.

El marco de referencia que ha de impregnar el tratamiento de estos problemas debe fundamentarse a partir de las posibilidades y recursos que debe ofrecer el contexto educativo. El derecho a ser educado incluye el deber de tratar este tipo de comportamientos desde perspectivas que permitan a unos y otros, agresores, víctimas y espectadores de uno u otro nivel de participación, que la experiencia sea reversible y de la que poder aprender. Las res- 
puestas al bullying deben planificarse con antelación. Aunque los modelos teóricos explicativos sirven como referencia a la hora de plantear estrategias de intervención, en muchas ocasiones éstas no están pensadas desde modelos estratégicos que incluyan a todo el sistema y organización escolar. Es posible que un centro tenga prevista alguna estrategia de intervención individual en situaciones puntuales de bullying, pero no tenga un sistema organizado y gradual de respuestas elaborado a partir de un enfoque teórico definido previamente. Ello explicaría por qué en un determinado centro pueden coexistir estrategias de mediación entre pares con reglamentos de funcionamiento de centro basados en planteamientos sancionadores de forma casi exclusiva.

Incluso teniendo en cuenta que algunos escolares con dificultades educativas y de integración social tienen mayor probabilidad que otros de verse implicados en algún tipo de relación abusiva con sus iguales, es importante que los centros elaboren estrategias de abordaje constructivas y educadoras que incluyan la reparación y la compensación por el daño causado.

\subsection{La implicación de los espectadores}

La intervención en el acoso escolar debe llevarse a cabo en y por la comunidad educativa implicando a todos sus miembros. Responsabilizarse de la seguridad del alumnado es una tarea del sistema educativo en general y de la dirección y del equipo de profesorado en particular. Esa responsabilidad debe traducirse en diversas medidas cuyo objetivo sea promover las relaciones sociales igualitarias entre el alumnado y favorecer las relaciones positivas. Velar por la seguridad del alumnado significa, en este caso, disponer de información sobre sus relaciones sociales ${ }^{2}$ e intervenir cuando éstas no sean adecuadas, sean deficitarias por aislamiento social o rechazo, o claramente dañinas como en el caso del acoso escolar.

En cuanto a la normativa del centro y del aula es importante crear expectativas positivas en las relaciones sociales entre el alumnado, así como crear y utilizar normas de comportamiento y formas de trabajo cooperativas. Los roles que se producen en una situación de bullying son un primer punto de partida para aproximarse a las formas de relación activas o pasivas en relación a estas conductas. Tal como hemos comentado, el bullying se produce en un contexto grupal de forma muy importante, con roles más comprometidos como el de agresor, el de víctima y el de los espectadores. Se puede precisar algo más esta primera aproximación en lo que Olweus (2001) denomina círculo del bullying. En el círculo, las posiciones pueden ir desde el agresor hasta el defensor de la víctima, el que secunda la agresión, el que muestra apoyo pero no interviene, los espectadores pasivos, los posibles defensores, etc.

La intervención en el ámbito grupal debe tener en cuenta estos roles en una situación de acoso. En muchas ocasiones, el alumnado, incluso teniendo actitudes positivas hacia la víctima, no las pone en práctica ante la fuerte presión que percibe para inhibirse a la hora de ofrecer ayuda a un compañero en una situación de dificultad (por miedo a represalias, por inseguridad en su intervención, por miedo al rechazo, etc.). De acuerdo con ello, se trata de ver cómo puede reducirse el bullying animando a los espectadores pasivos a implicarse e intervenir, llevando a cabo conductas prosociales para ayudar a sus compañeros víctimas de bullying.

Es necesario comprender los diversos roles activos o pasivos de los observadores en las situaciones de bullying, si bien se debe tener en cuenta que la deseabilidad social juega un papel muy importante. Al 
respecto, se ha estudiado el rol de los observadores (si están presentes o no cuando se produce la situación de bullying, el tipo de respuestas que dan y en qué forma) utilizando diversas metodologías, algunas de ellas con menor riesgo de sesgo desde el punto de vista de la deseabilidad social que otras.

La presencia de los espectadores en los episodios de acoso es muy elevada. Diversos estudios de prevalencia en presencia de observadores utilizando métodos de observación directos y empleando videocámaras y micrófonos sin hilos (Pepler y Craig, 1995, en Rigby y Johnson, 2006) informan de que los iguales entre 5 y 12 años estaban presentes en el $85 \%$ de los episodios de bullying en el patio escolar.

En el caso de las respuestas de los observadores, y de acuerdo con los datos de diversos estudios, por ejemplo, O'Connell et als., (1999) utilizando grabaciones de vídeo, los resultados indicaron que, partiendo del $100 \%$ total de presencia del observador, sólo un $25,4 \%$ del tiempo se empleó en desanimar la agresión. El resto del tiempo se utilizó o en animar al agresor, el 20,7\%, o en reforzar el bullying de forma pasiva observando sin formar parte en el 53\%. Así pues, los observadores pueden ser de varios tipos: asisten y ayudan físicamente al agresor; refuerzan incitando a la violencia y a la exclusión; observan neutrales e inactivos pretendiendo no ver nada, y muy pocos defienden ayudando a la víctima y haciendo frente al agresor (Salmivalli et als., 1996). A pesar de ello, la mayoría de los observadores desaprueban estos comportamientos, y a una parte importante de ellos les gustaría hacer algo para ayudar (Schulman, 2002). Sin embargo, más de la mitad de las intervenciones llevadas a cabo para desanimar las acciones de bullying fueron efectivas para lograrlo (Hawkins et als., 2001, en Rigby y Johnson, 2006). Otra forma de evaluar la conducta de los observadores en las escuelas es utilizando cuestionarios de evaluación en los que se pregunta a los estudiantes cómo responden sus compañeros cuando observan situaciones de bullying. Salmivalli et als., (1997) utilizaron este método en Finlandia con 459 escolares entre 11 y 12 años. De acuerdo con sus resultados, sólo el 16,5\% de los estudiantes defendían a las víctimas.

Sin embargo, cuando se utilizan medidas de evaluación como el autoinforme, el porcentaje de comportamientos de ayuda a las víctimas del que se informa en las respuestas de los estudiantes de primaria es mucho más elevado: entre el $43 \%$ y el $67 \%$ según diversos estudios. Los porcentajes de comportamientos de ayuda pueden ser incluso más elevados cuando al alumnado se le pide que informe de su comportamiento precisando en las distintas formas de maltrato (Rigby y Johnson, 2006; Orte et als., 1997-2000).

El contraste entre estimaciones basadas en datos observacionales directos y estimaciones de autoinformes sugiere que estos últimos pueden estar sesgados por respuestas deseables socialmente. Así pues, parece claro que las respuestas de ayuda de los iguales en situaciones de bullying son más bien escasas y menos frecuentes a medida que aumenta la edad. Otras variables que tienen interés para precisar algo más las conductas de ayuda de quienes defienden a las víctimas son la amistosidad, las actitudes positivas hacia las víctimas, la confianza en la habilidad de uno mismo en influir en los resultados de forma positiva o autoeficacia, la presión normativa del grupo de pares en el contexto de la conducta en la escuela (Rigby y Johnson, 2006) y la protección de los amigos (Boulton et als., 1999). Una forma adecuada de extender esta protección más allá del círculo de amigos es creando contextos posibilitadores de estos comportamientos positivos. 


\section{Implicaciones para la intervención}

\subsection{En los centros educativos}

La intervención en los problemas de acoso escolar debe concretarse en cada realidad conociendo el problema y pensando soluciones colectivas que impliquen a toda la comunidad educativa. A partir de la información proporcionada por el alumnado, que puede completarse con la del profesorado y la de los padres, se puede adecuar la intervención a cada contexto. Ya se ha comentado la importancia de pensar en intervenciones que se dirijan al alumnado como conjunto, que es quien tiene la capacidad para mantener, aumentar o disminuir estos comportamientos. El centro educativo puede contribuir en la mejora de las relaciones interpersonales fomentando la empatía y las relaciones positivas entre los grupos de alumnado, creando expectativas positivas de comportamientos de ayuda y formando al alumnado en respuestas de ayuda que permitan reforzar la autoeficacia personal en estas situaciones de acoso.

La intervención con los observadores es una cuestión prioritaria en el conjunto de las acciones que se lleven a cabo. Se deben poner en marcha programas cuyo objetivo sea motivar a los observadores para que ayuden a las víctimas. Los puntos en los que puede concretarse el trabajo a llevar a cabo invirtiendo los recursos en los observadores incluyen, en primer lugar, formar al profesorado en estrategias de diagnóstico y de intervención grupal en el aula teniendo en cuenta, por un lado, que el acoso escolar se produce delante de los observadores y que el profesorado rara vez es informado por éstos. Los puntos más importantes a llevar a cabo por parte del profesorado, son los siguientes (Rigby y Johnson, 2004):

1. Ayudar al alumnado a formular y expresar su propia forma de ver cómo deben comportarse los observadores, ayudándoles en la adquisición de las habilidades necesarias para poder ser de ayuda.

2. Animar a la expresión de respuestas empáticas hacia las víctimas.

3. Animar al alumnado a llevar a cabo comportamientos de ayuda y reforzarlos.

4. Enseñar al alumnado formas constructivas de actuación cuando observen comportamientos de acoso en el centro. Siguiendo la propuesta de Rigby $(2003 \mathrm{~b})$, mediante discusiones abiertas sobre el comportamiento de los observadores ante distintas situaciones visualizadas a través de dibujos, fotos, películas, etc.

5. Identificar y debatir sobre aquellas situaciones en las que el alumnado esté de acuerdo en que intervenir puede ser peligroso, enseñándoles a pedir ayuda a otros.

6. Trabajar con el alumnado sobre las formas en las que el riesgo de intervenir pueda minimizarse, por ejemplo, haciendo afirmaciones que muestren que a uno le disgusta ese comportamiento, en lugar de implicarse físicamente en cualquier pelea, y animando a otros observadores a verbalizar también su oposición.

7. Ensayar con los estudiantes lo que deben decir cuando vean que el bullying tiene lugar llevando a cabo estrategias de juego de rol en situaciones simuladas como observadores.

8. Animar al alumnado a contar a la clase sus experiencias como observadores cuando hayan respondido de forma positiva en situaciones de bullying. Comentar con el alumnado los resultados de su conducta, reforzarla positivamente y explorar las dificultades y las posibles soluciones.

Este tipo de contenidos deben formar parte de estrategias más amplias de trabajo en las que se enseñe a los estudiantes a mostrar su desaprobación, contribuyendo a fomentar respuestas de ayuda entre iguales ante las agresiones. Con esa finalidad se ha desarrollado y evaluado (Cowie, 2000; Naylor y Cowie, 1999; Cowie y Hutson, 2005) un conjunto de técnicas grupales en 
la misma línea comentada, pensadas para desarrollar el potencial de apoyo y ayuda del alumnado que, aunque no está de acuerdo con los agresores, no interviene para ayudar a las víctimas. En este contexto $y$ de forma estructurada, se forma $y$ apoya a este alumnado para que pueda ofrecer distintos tipos de apoyo, ayuda y colaboración a otros iguales en situaciones de vulnerabilidad y dificultad como es el caso del bullying.

En lo que se refiere a las características principales de estas acciones de ayuda entre iguales, se forma al alumnado para trabajar juntos fuera de su círculo de amistad. Este tipo de interacción ayuda a reducir los prejuicios y fomenta la confianza entre personas de distinto sexo y grupo cultural de pertenencia. A través de una formación adecuada, se proporciona al alumnado la oportunidad de aprender habilidades de comunicación, compartir información y reflexionar sobre sus emociones. Se forma al alumnado para tratar con los conflictos y para ayudar a sus compañeros a relacionarse los unos con los otros de una forma más constructiva (Cowie y Wallace, 2000), como proporcionar amistad o apoyo en la interacción diaria, aportar modelos de referencia y de guía positivos, consejo e información en temas escolares o no escolares de interés para este alumnado. En definitiva, el apoyo social necesario estructural y funcionalmente, para que el alumnado víctima de bullying participe de las redes sociales de ayuda, mejore la calidad de sus relaciones con los iguales y poder prevenir y/o reducir el impacto de las conductas de bullying. El tipo de apoyo y ayuda es de distinto tipo y se utiliza de un modo u otro dependiendo del nivel educativo -primaria o secundaria-, y del objetivo. Así, por ejemplo, un objetivo puede ser detectar y ayudar a estudiantes que están solos en el aula y el patio de recreo o ayudar al alumnado que tiene problemas de adaptación, o aplicar la solución de conflictos in- terpersonales en conflictos planteados por el alumnado más mayor.

Los principales tipos de ayuda entre iguales son los siguientes:

- La hora del círculo: con una frecuencia semanal, el alumnado comparte sus preocupaciones y sentimientos con los demás y, si es posible, busca alternativas para mejorar la situación.

- Compañeros amigos: voluntarios de la misma edad o mayores, entrenados en habilidades interpersonales, prestan su ayuda y amistad a otros estudiantes en riesgo de victimización.

- Mediación y resolución de conflictos: estudiantes formados en técnicas de mediación y solución de conflictos ayudan a otros estudiantes a encontrar una solución satisfactoria para ambos ante diferentes conflictos.

- Escucha activa: estudiantes formados en esta técnica ayudan a otros estudiantes en situaciones de malestar en un contexto formalizado y supervisado.

- Tutorización de iguales: estudiantes más mayores formados para este fin guían y modelan a otros más jóvenes mediante el contacto individual y a través de juegos, ayuda en los deberes y escucha en los problemas y preocupaciones.

Las ventajas de la ayuda entre iguales, de acuerdo con los trabajos al respecto de Cowie y Hutson (2005), son las siguientes:

1. El alumnado detecta la violencia en las fases más tempranas.

2. Es más probable que el alumnado confíe en un igual más que en un adulto.

3. Las victimas tienen a alguien a quien acudir y perciben que en la escuela se actúa en el problema.

4. Supone una ayuda también para el profesorado, a quien a veces falta tiempo y recursos para ocuparse de todos los problemas interpersonales que le llegan. 
5. El alumnado formado como ayudante aprende habilidades interpersonales que le serán de mucho valor en su propio ejercicio de la ciudadanía activa.

6. Los padres también visualizan y valoran la preocupación de la escuela por el bienestar de los estudiantes.

7. Los sistemas de ayuda ofrecen relaciones potenciales con otros servicios, por ejemplo, los departamentos de orientación y otros recursos dentro o fuera del centro.

8. Entre los ayudantes, a menudo están quienes fueron víctimas de violencia. Esto les hace tener mayor empatía por la experiencia e integrarse en grupos en los que predominan el apoyo y la ayuda.

\subsection{En la comunidad. El rol del educador social}

Antes de referirme al rol del educador social en los problemas de bullying escolar enunciados anteriormente, quizás convenga preguntarse si la educación social tiene algún rol en la institución escolar y si debería tenerlo. La formación de los educadores sociales en relación a la institución escolar no ha tenido ningún protagonismo, excepto de forma indirecta y tangencial, por coincidir con la edad de escolarización de los grupos con los que se esté trabajando en otros programas fuera de la escuela.

Sin embargo, los cambios sociales, la existencia creciente de la inmigración, los cambios culturales, las nuevas realidades familiares, la evolución demográfica, la existencia de nuevas necesidades sociales, las nuevas realidades urbanas, el trabajo de los servicios sociales y culturales, la nueva cultura del ocio urbano, la transformación del sistema educativo, etc., plantean nuevas demandas en las que la educación social tiene, incluso sin apenas habérselo planteado, un protagonismo cada vez mayor.

De hecho, y pese a su carácter minoritario, se han empezado a desarrollar pro- gramas socioeducativos por parte de los educadores sociales con la institución escolar como objetivo. Fundamentalmente, se trata de programas dirigidos a la resolución de problemas y de conflictos que se manifiestan en el seno de la institución escolar y para los que algunos de los profesionales con los que ésta cuenta creen no tener respuestas, más allá de la aplicación del reglamento de régimen interno. Es por esta razón por la que cada vez más se solicita la intervención del profesional de la educación social. Esta colaboración ya se había dado en muchos casos por la vía del trabajo en red desde otros servicios, fundamentalmente servicios sociales, servicios de protección al menor, servicios de reforma, centros de ocio y tiempo libre, etc. En otros casos sin embargo, se solicita su incorporación a los centros escolares para ocuparse de problemas sociales concretos. En este sentido hace falta hablar, a título de ejemplo, de los programas en relación al absentismo escolar, a los problemas de convivencia educativa, al bullying escolar, al maltrato infantil, a las infracciones contra las normas, a la drogadicción o al fracaso escolar. Incluso empieza a haber normativa por parte de algunas comunidades autónomas en la que se regula la participación del educador social en el seno de las instituciones escolares.

Se trata de algunos indicios que muestran las posibles tendencias de futuro en la institucionalización de nuevas funciones para el educador social y sobre las que hay que reflexionar. La educación social, efectivamente, tiene un rol en la institución escolar, y por ello se deben hacer proyectos de intervención socioeducativa dirigidos a potenciar la socialización positiva, la competencia social, la inclusión social del alumnado en los centros educativos, algunos de los cuales ya son miembros de otros centros y de otros grupos, por presentar dificultades sociales. La dimensión social 
de la educación es un objetivo apenas asumido, apenas esbozado en la institución escolar, que sí puede asumirse desde la educación social. El profesional de la educación social puede cumplir muy bien con ese cometido; eso sí, poniendo en marcha proyectos de intervención socioeducativa no sólo en situaciones de inadaptación, sino también de normalidad y de inclusión. No sólo en programas de tratamiento, sino también y, fundamentalmente, en programas de prevención.

Desde nuestro punto de vista, es necesario ubicar al profesional de la educación social en la institución escolar desde el trabajo comunitario, desde el trabajo realizado desde los servicios sociales de asistencia primaria, desde la proximidad. No se trata de negar la posibilidad de contratar educadores sociales en los centros educativos, aunque pensamos que resulta mucho más adecuado el trabajo del educador social más integrado, más plural desde la perspectiva funcional, más en equipo, más compactado. Esta es la línea a seguir si se quiere contribuir a construir una figura profesional desde la normalidad y la inclusión social.

Así pues, el trabajo, las nuevas funciones, las nuevas competencias del educador social dentro de este campo escolar, deben entender la institución escolar como un espacio estratégico en el proceso de posibilitar la integración y la inserción social de las jóvenes generaciones, que en su totalidad pasan por la escuela como espacio de socialización, de formación o de educación, desde la normalidad. La condición sobre la que es necesario reflexionar es la reconsideración de la institución escolar de acuerdo con los retos que la sociedad le plantea a la comunidad escolar. Unos retos en los que no sólo es importante el conocimiento, sino también el desarrollo de las competencias y las habilidades sociales necesarias para la integración y la convivencia dentro de la sociedad, con todo lo que este hecho implica de conocimiento y de comprensión de la realidad actual, de los retos, de las oportunidades y de las dificultades que plantea el siglo actual, de acuerdo con su complejidad creciente y cambiante.

De acuerdo con ello, la educación social debe formar parte de los objetivos educativos de la institución escolar. La escuela actual debe ser capaz de conocer y comprender los retos de la sociedad del presente y del futuro, de contribuir a la socialización de los alumnos de hoy, de adaptarse a los cambios habidos en las estructuras familiares, de asimilar las nuevas tecnologías, de comprender los movimientos migratorios, de insertarse dentro de la sociedad del conocimiento de forma plena.

En la actualidad, el refuerzo del papel inclusivo de la educación resulta fundamental. En la consecución de este objetivo, la educación social, incardinada en la institución escolar, debe jugar un papel primordial en la institucionalización de una nueva justicia y equidad social. Se trata de una nueva concepción de la escuela y de la educación social, con una nueva relación con la familia, los servicios sociales y todo el tejido social. Se trata de una nueva concepción de la Pedagogía Social que incluya la institución escolar en su conceptualización, en su investigación, en su profesionalización y en su praxis cotidiana (March y Orte, 2007a, 2007b).

Una vez en la institución escolar, el trabajo del educador social cobra sentido en relación a los problemas de acoso e intimidación en la escuela. Estos problemas deben abordarse teniendo en cuenta todos los recursos y sistemas organizativos de que disponen los centros. Con todo, la dimensión social de la educación es un objetivo a veces inexistente en la escuela, fundamentalmente a partir de la educación primaria. Alcanzar este objetivo significa llevar a cabo actuaciones que en muchos casos forman 
parte del bagaje formativo del educador social. Así, aunque el perfil del educador social no sea el más importante en la intervención en estos problemas en este marco, puede prestar su apoyo tanto en los proyectos normalizados de atención individual y grupal cuyo objetivo sea la mejora de la socialización como estrategia preventiva, como en aquellos programas que haya que llevar a cabo con el alumnado agresor y con el alumnado víctima; perfiles de alumnado que muchas veces requieren un tratamiento más individualizado para controlar el comportamiento, la empatía y las habilidades sociales, y para los que el profesorado y las familias necesitan un apoyo social específico.

Además de la ayuda y el asesoramiento a las familias, el diseño de proyectos educativos individualizados en los que la mejora de las relaciones sociales sea el principal objetivo, el trabajo en red con el centro y con otras instituciones comunitarias. Enfrentarse a los problemas de bullying requiere un enfoque holístico desde varios ámbitos. Supone un acercamiento global escuela-comunidad que implique a las familias de forma activa, mejore las relaciones personales y recupere los vínculos comunitarios. La intervención en el bullying no puede asumirse sólo por los centros educativos. Es necesaria una visión ecológica y un compromiso amplio y unánime de los diversos agentes sociales. Esto puede llevarse a cabo a través de programas pensados para las familias que impliquen al profesorado y a otros agentes sociales de los recursos comunitarios como los servicios sociales, de salud, de ocio y tiempo libre, así como los recursos especializados en menores. Se trata de lograr, mediante distintos medios, que el trabajo que se está llevando a cabo en los centros pueda tener mayor continuidad, apoyo, formación, convergencia de mensajes y contenidos educativos. Es en este contexto en el que el rol y la competencia del educador social pueden tener protagonismo en la continuidad de estos programas; implementando los contenidos a los que se ha hecho referencia y ejerciendo el papel de mediador y coordinador del conjunto de acciones y programas que se pueden llevar a cabo por diferentes instituciones de la comunidad en relación al bullying.

\section{Referencias bibliográficas}

Batsche, G. M. y Knoff, H. M. (1994): "Bullies and their victims: understanding a pervasive problem in the schools", en School Psychology Review, 23, 2, pp. 165-174.

Boulton, M. J. y Underwood, K. (1992): "Bully/victim problems among middle school children", en British Journal of Educational Psychology, 62, pp. 73-87.

Boulton, M. J.; Trueman, M.; Chau, C.; Whitehand C. y Amatya, K. (1999): "Concurrent and longitudinal links between friendship and peer victimization: implications for befriending interventions", en Journal of Adolescence, 22, pp. 461-466.

Cowie, H. (1999): "Bystanding or standing by: gender issues in coping with bullying in english schools”, en Aggressive Behavior, 26, pp. 85-97.

Cowie, H. y Hutson, N. (2005): "Peer support: a strategy to help bystanders challenge school bullying", en Pastoral Care, pp. 40-44.

Cowie, H. y Wallace, P. (2000): Peer support in action. London: Sage.

Defensor del Pueblo (2007): Violencia escolar: El maltrato entre iguales en la educación secundaria obligatoria 1999-2006. Madrid: Autor.

Maines, B. y Robinson, G. (1992): Michael's Story: The "No Blame" Approach. Bristol: Lame Duck Publishing.

March, M. X. y Orte, C. (2007a): "L'educació social i la institució escolar: la recuperación del treball amb l'escola per part de l'educador social", en Trobada d'Educació Social. Revista del CEE$S I B, 1$, pp. 14-15.

March, M. X. y Orte, C. (2007b): "El trabajo interdisciplinar del educador social en la escuela", en Aula de Innovación Educativa, 160, pp. 12-14. 
Menesini, E.; Codecasa, E., y Benelli, B. (2003): “Enhancing children's responsibility to take action against bullying: Evaluation of a befriending intervention in Italian middle schools", en Aggressive Behavior, 29, pp. 10-14.

Naylor, P. y Cowie, H. (1999): "The effectiveness of peer support systems in challenging school bullying: the perspectives and experiences of teachers and pupils", en Journal of Adolescence, 224, pp. 467-479.

O'Connell, P.; Pepler, D. y Craig, W. (1999): “Peer involvement in bullying: Insights and challenges for intervention", en Journal of Adolescence, 22, pp. $437-45^{2}$.

Olweus, D. (1993): Bullying in Schools. Cambridge, Massachusetts: Blackwell.

Olweus, D. (2001): "Peer harassment. A critical analysis and some important issues", en Juvonen, J. S. y Graham (eds.): Peer harassment in school (3-20). Nueva York: The Guildford Press.

Orte, C. (2002): Prevención de la violencia de género en la Educación Secundaria Obligatoria. Congreso Interdisciplinar sobre Educación y Género. Málaga: Universidad de Málaga.

Orte, C. (2005): "Bullying: la necesidad del abordaje multidisciplinar", en Formación Médica Continuada en Atención Primaria, 12, 3, pp. 130-138.

Orte, C. (2006a): "Nuevas perspectivas sobre la violencia y el bullying escolar", en Panorama Social, 3, pp. 27- 41.

Orte, C. (2006b): Educación familiar y prevención de la violencia de género. XX Seminario Interuniversitario de Pedagogía Social. "Educación social e igualdad de género", Málaga: Universidad de Málaga.

Orte, C. y March, M. X. (1996): "El bullying versus el respeto a los derechos de los menores en la educación: La escuela como espacio de disocialización", en Pedagogía Social. Revista Interuniversitaria, 14, pp. 47-62.

Orte, C.; March, M. X.; Ballester, L. y Ferrà, P. (1997-2000): El maltrato e intimidación entre iguales, "bullying", en el medio educativo. Madrid, Dirección General de Enseñanza Superior [Número: PB96-0192].

Orte, C.; Touza, C.; Ballester, L. y Ferrà, P. (2004): "Bullying, els nens es maltracten. Diferències de gènere, victimització i agressió escolar", en Tres
Quarts. Revista Especialitzada en Joventut i Lleure, 17, pp. 14-19.

Pikas, A. (2002): "New developments of Shared Concern Method", en School Psychology International, 23, pp. 307-326.

Rigby, K. (2002): New Perspectives on Bullying. Londres: Jessica Kingsley.

Rigby, K. (2003a): Addressing bullying in schools: Theory and Practice, Trends and Issues. Australian Institute of Criminology [fecha de acceso: 29 de marzo de 2007], URL disponible en: http://www.aic.gov.au/publications/tandi2/tandi2 59.html.

Rigby, K. (2003b): Stop the bullying: a handbook for schools. Melbourne: ACER.

Rigby, K. y Johnson, B. (2006): "Expressed readiness of Australian school children to act as bystanders in support of children who are being bullied", en Educational Psychology, 26, 3, pp. 425-440.

Rigby, K. y Johnson, B. (2004): "Innocent bystanders?”, en Teacher, pp. 38-40.

Salmivalli, C. (1999): "Participant role approach to school bullying: Implications for interventions", en Journal of Adolescence, 22, pp. 453-459.

Salmivalli, C.; Huttunen, A. y Lagerspetz, K. M. (1997): "Peer networks and bullying in schools", en Scandinavian Journal of Psychology, 38, pp. 305-331.

Salmivalli, C.; Lagerspetz, K.; Björkqvist, K.; Osterman, K. y Kaukiainen, A. (1996): "Bullying as a group process: participant roles and their relation to social status within the group", en Aggresive Behavior, 22, pp. 1-15.

Schulman, M.: (2002). "How we become moral", en Zinder C. R. y López, D. L. (eds.) Handbook of positive psychology (499-512). Oxford: Oxford University Press.

Slee, P. T. y Rigby, K. (1993): “The relationship of Eysenck'spersonality factors and self-esteem to bully/victim behaviour in Australian schoolboys", en Personality and Individual Differences, 14, pp. 371-73.

Smith, P. K.; Morita, Y.; Junger-Tas, J.; Olweus, D.; Catalano, R. y Slee, P. (eds.) (1999): The nature of school bullying: A cross-national perspective. London: Routledge. 
Smith, P. K., y Sharp, S. (eds.) (1994): School Bullying: Insights and Perspectives. Londres: Routledge.

Society for Prevention Research (2007): 15th Annual Meeting. Advancing Science-Based Prevention. Creating Real World Solutions. Washington, DC.

Soutter, A. y McKenzie, A. (2000): "The use and effects of anti-bullying and anti-harassment policies in Australian schools", en School Psychology International, 21 (2000), pp. 96-105.

\section{Notas}

${ }^{1}$ Digamos que normal o natural no tiene en cuenta el daño a la víctima. Es decir, de lo que se entiende que una persona puede aceptar o incluso "soportar", sin sufrir más de lo necesario; o en todo caso, sin que ese sufrimiento produzca el daño suficiente como para ocasionar problemas psicológicos o incluso psiquiátricos importantes a corto, medio y largo plazo. Se trata más bien de un planteamiento centrado en la ejecución de la agresión sin más, o en las razones que pueden llevar a un niño o niña a producir este tipo de acciones a otros.

${ }^{2}$ El alumnado es quien mejor conoce la realidad de su propio grupo. Técnicas como la nominación entre iguales, para conocer al alumnado implicado en situaciones de maltrato y el sociograma, para conocer y clasificar al alumnado según su estatus sociométrico, son buenas opciones de diagnóstico para conocer las relaciones del grupo.

DiReCCiÓn DE LA AUTORA: Carmen Orte Socias. Universitat de les Illes Balears. Departamento de Pedagogía y de Didácticas Específicas. Carretera de Valldemossa, km. 7,5. Campus Universitario. Edificio Guillem Cifre de Clonya. 07122 Palma (Mallorca). Correo electrónico: carmen.orte@uib.es

Fecha de recepción del artículo: 10.XI.2007

Fecha de aceptación definitiva: 20.XII.2007 
